\title{
Compressive Color Pattern Detection using Partial Orthogonal Circulant Sensing Matrix
}

\author{
Sylvain Rousseau*, David Helbert ${ }^{\dagger}$ \\ *Heudiasyc Laboratory, CNRS U-7253, University of Technology of Compiègne, France \\ ${ }^{\dagger}$ Xlim-ASALI, CNRS U-7252, University of Poitiers, France
}

\begin{abstract}
One key issue in compressive sensing is to design a sensing matrix that is random enough to have a good signal reconstruction quality and that also enjoys some desirable properties such that orthogonality or being circulant. The classic method to construct such sensing matrices is to first generate a full orthogonal circulant matrix and then select only a few rows. In this paper, we propose a refined construction of orthogonal circulant sensing matrices that generates a circulant matrix where only a given subset of its rows are orthogonal. That way, the generation method is a lot less constrained leading to better sensing matrices and we still have the desired properties. The proposed partial shift-orthogonal sensing matrix is compared to random and learned sensing matrices in the frame of signal reconstruction. This sensing matrix is pattern-dependent and thus efficient to detect color patterns and edges from the measurements of a color image.
\end{abstract}

\section{INTRODUCTION}

To face the constantly growing amount of data coming from sensors, a new acquisition model has recently emerged [1], [2], [3], [4], [5]. Described in the seminal work of Candès, Romberg and Tao in [6] and Donoho in [7], compressive sensing aims at performing the acquisition and compression steps of the classical acquisition paradigm in one single step. This step consists of taking a limited number $m$ of linear measurements on a signal $x$ (1D signal or digital images) of length $n$.

$$
y_{i}=\left\langle\phi_{i}, x\right\rangle, \quad i=1, \ldots, m,
$$

where $m$ is much smaller than $n$ or, in short, $y=\mathbf{\Phi} x$, where $\Phi$ is referred to as the sensing matrix.

Recovering $x$ from $y$ is an ill-posed problem but if the signal $x$ is sparse in some known basis or dictionary $\boldsymbol{\Psi}$, meaning that $x=\mathbf{\Psi} u$ with $u$ sparse, the following minimization problem is able to recover $x$.

$$
\underset{u}{\arg \min }\|u\|_{1} \quad \text { s.t. } \quad \boldsymbol{\Phi} \mathbf{\Psi} u=y .
$$

The matrix $\Psi$ will be referred to as the sparsifying basis or dictionary.

For minimization (1) to successfully recover $x$, the sensing matrix $\Psi$ has to be carefully chosen. It has been shown that random measurement taking is optimal in some sense [6]. Although we have theoretical guarantees that minimization (1) will succeed, random measurements prevent minimization problem (1) from being efficiently solved. Indeed, the key for most algorithms is to be able to quickly calculate products by the sensing matrix $\boldsymbol{\Phi}$ and its transpose. We somehow have to design deterministic sensing matrices to deal with larger signal size. One possible solution is to choose the sensing matrix as a fast transform followed by a projection [8]. However, these measurement taking processes are not very realistic when it comes to implementing them in a sensor. A more realistic one is proposed in [9], [10] where sensing matrices are orthogonal and circulant or Toeplitz. These sensing matrices are both physically realistic as a convolution operator, easy to multiply with only two FFTs and offer comparable recovering properties [11], [12], [13].

To measure the quality of a sensing matrix, several metrics have been proposed. The restricted isometry property (RIP) proposed in [14] gives a measure quantifying how well the sensing matrix is mapping isometrically signals of interest to measurements. Another metric called mutual coherence between the sensing matrix and the sparsifying dictionary has been introduced in [15] and is easier to compute. Using this metric, contributions have been proposed to learn both the sparsifying dictionary and the sensing matrix from the signals of interest [16] or requiring that the sensing matrix is circulant [17]. In [18], the sensing matrix is designed by minimizing its mutual coherence with a given sparsifying basis.

Template detection in a multispectral image is one of the first application when dealing with multispectral data. It consists of locating a template within a multispectral image. Several algorithms have been proposed so far to tackle down this problem, see [19] and references therein. In our previous paper [20], we have adapted this minimization problem to the compressive sensing and proved that we can detect a multispectral pattern from a compressive sensing of a color image. However it is more difficult to detect patterns on an image with few bands as a color image and we thus propose to generate a partial shift-orthogonal matrix, a pattern-dependent circulant matrix whose only rows we are interested in form an orthogonal family.

The outline of this paper is as follows. Section II introduces the principle of the compressive pattern detection. Section III recalls what a shift-orthogonal matrix is and defines all the necessary definitions. It is shown how to generate less restrictive shift-orthogonal matrices called partial shift-orthogonal matrices. Section IV is devoted to numerical experiments comparing the efficiency of sensing matrices with synthetic and magnetic resonance spectroscopy signals. Section V then presents and discusses results in the frame of detections of color patterns and edges in the compressive sensing of color images. Section VI concludes the paper. 


\section{COMPRESSIVE SPATIAL PATTERN RECOGNITION}

In a previous work, we proposed a method to detect a spectral signature [21] in a compressive hyperspectral sensing context and extented it for the detection of a spatial pattern [20]. In this section we recall both methods.

\section{A. Template detection}

Detecting a particular signature in a multispectral image is a fundamental task in multispectral imaging. It consists in finding the locations of a given signature called a template.

In [19], Guo and Osher suggested the following minimization problem that leverages the sparsity inducing ability of the $\ell_{1}$ norm to find those locations.

$$
\underset{u \geq 0}{\arg \min }\|u\|_{1} \quad \text { s.t. } \quad\left\|\boldsymbol{X}^{T} u-s\right\|_{2}<\sigma .
$$

The vector $s$ denotes the template we want to detect and $\boldsymbol{X}$ the multispectral image where each column corresponds to a channel and each row is the spectrum of a pixel.

In [21], we proposed a minimization problem in a compressive sensing context where we only have access to a small number of linear measurements on the multispectral image. We proposed to reconstruct the vector $u$ solution of (2) without reconstructing the image first. The acquisition model is then described as

$$
\boldsymbol{M}=\boldsymbol{F} \boldsymbol{X},
$$

where $\boldsymbol{F}$ is a sensing matrix and we showed that the compressive template matching can be solved by the following minimization:

$$
\underset{u \geq 0}{\arg \min }\|u\|_{1} \quad \text { s.t. } \quad\left\|\boldsymbol{M}^{T}\left(\boldsymbol{F} \boldsymbol{F}^{T}\right)^{-1} \boldsymbol{F} u-s\right\|_{2}<\sigma .
$$

The multispectral image $\boldsymbol{X}$ is replaced in (2) by the closest matrix w.r.t the Frobenius norm such that $\boldsymbol{M}=\boldsymbol{F} \boldsymbol{X}$.

\section{B. Spatial pattern detection}

We extended the compressive template detection minimization problem to perform compressive spatial pattern detection with a special transform called spectralization. We present in this section the core principle, the complete mathematical formalism can be found in [20].

Suppose we want to detect of spatial pattern described by the following shape

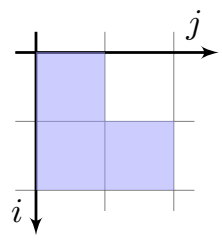

This spatial pattern $P$ is encoded as an ordered list of couples

$$
P=((0,0),(1,0),(1,1)) .
$$

The spectralization of a grayscale image $\boldsymbol{I}$ along $P$, denoted $\operatorname{spec}_{P}(\boldsymbol{I})$ is the process of adding shifted copies of $\boldsymbol{I}$ as new spectral bands. Shifts are controlled by the shape of the spatial pattern $P$. The spectralization of a grayscale image along the pattern $P$ is depicted in Figure 1. It allows us to recast a complex spatial pattern detection into a simpler template detection.

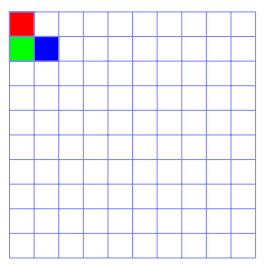

(a) Grayscale image

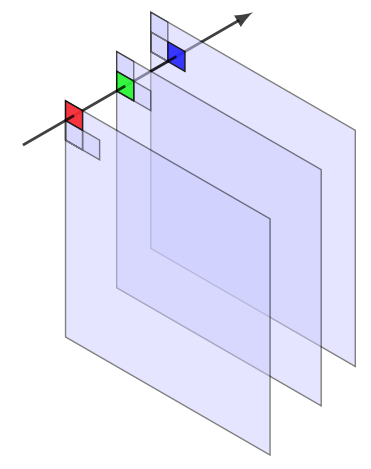

(b) Spectralization along the pattern (6)
Fig. 1: Spectralization of a grayscale image along the hook pattern

\section{Partial ShifT-Orthogonal SEnSing Matrices}

Both compressive spatial pattern detection and compressive template detection rely on minimization (4). We then need to choose $\boldsymbol{F}$ such that $\left(\boldsymbol{F} \boldsymbol{F}^{T}\right)^{-1}$ is easily computable. One way to do so is to choose $\boldsymbol{F}$ to have orthogonal rows.

\section{A. Shift-orthogonal matrices}

The spectralization also imposes us to have measurement vectors (rows of $\boldsymbol{F}$ ) to be shifted versions from one another once they are reshaped to match the image width and height (see [20]). Here we only consider the reshaped version of one measurement vector and call it the measurement matrix, not to be confused with the sensing matrix. A measurement matrix whose shifted copies form an orthogonal family will be called a shift-orthogonal matrix.

In the following, before introducing partial shift-orthogonal matrices, we first make a few definitions and assumptions. For the sake of simplicity, we use 0-based matrix coordinates. Given an element $p=(i, j)$ of $\mathbb{Z}^{2}$, we define the operator $S_{p}$ acting on a matrix that shifts all its entries by $p$. More formally, if $\boldsymbol{A}$ is of size $N \times M$ we have

$$
\left[S_{p}(\boldsymbol{A})\right]_{a b}=\boldsymbol{I}_{(a+i \bmod N),(b+j \bmod M)} .
$$

The purpose of this paper is to find a measurement matrix $\boldsymbol{A}$ given a set of shifting vectors $E=\left\{e_{1}, \ldots, e_{|E|}\right\} \subset \mathbb{Z}^{2}$ such that the set of matrices

$$
S_{e_{1}}(\boldsymbol{A}), S_{e_{2}}(\boldsymbol{A}), \ldots, S_{e_{|E|}}(\boldsymbol{A}),
$$

once vectorized, form an orthogonal family.

To that end, we introduce a few operators acting on matrices. First, the correlation of two matrices is denoted by the operator $\circledast$. Formally, it is defined as follows.

$$
(\boldsymbol{A} \circledast \boldsymbol{B})_{i j}=\operatorname{Tr} S_{(i, j)}(\boldsymbol{A})^{T} \boldsymbol{B} .
$$


The upper left coefficient stores the inner product of the two matrices $\boldsymbol{A}$ and $\boldsymbol{B}$. The other coefficients are inner products of a shifted copy of $\boldsymbol{A}$ with $\boldsymbol{B}$. For example, if we have the two following matrices $\boldsymbol{A}$ and $\boldsymbol{B}$

$$
\boldsymbol{A}=\left(\begin{array}{lll}
3 & 1 & 2 \\
3 & 3 & 1
\end{array}\right) \quad \text { and } \quad \boldsymbol{B}=\left(\begin{array}{lll}
1 & 3 & 1 \\
2 & 3 & 3
\end{array}\right)
$$

Their correlation $\boldsymbol{C}$, denoted $\boldsymbol{A} \circledast \boldsymbol{B}$, is then

$$
\boldsymbol{C}=\left(\begin{array}{lll}
26 & 32 & 28 \\
28 & 29 & 26
\end{array}\right) \text {. }
$$

Second, the well-known Fourier transform of a matrix is denoted by the operator $\mathscr{F}$. And last, the operator denoted by $\mathscr{R}$ is defined as follows.

$$
(\mathscr{R} \boldsymbol{A})_{i j}=\boldsymbol{A}_{-i,-j} .
$$

With the previous example, we have

$$
\mathscr{R} \boldsymbol{A}=\left(\begin{array}{lll}
3 & 2 & 1 \\
3 & 1 & 3
\end{array}\right) \quad \text { and } \quad \mathscr{R} \boldsymbol{B}=\left(\begin{array}{lll}
1 & 1 & 3 \\
2 & 3 & 3
\end{array}\right)
$$

It is related to the Fourier transform by the following identity.

$$
N M \mathscr{R}=\mathscr{F}^{2},
$$

which shows in particular that $\mathscr{F}$ and $\mathscr{R}$ commute. Another link between the two operators is that $\mathscr{R}$ characterizes the matrices that have a Fourier transform that is real. More precisely, $\boldsymbol{A}$ is real if and only if $\mathscr{F} \boldsymbol{A}$ is $\overline{\mathscr{R}}$-invariant, which means that

$$
\overline{\mathscr{R}} \mathscr{F} \boldsymbol{A}=\mathscr{F} \boldsymbol{A},
$$

with $\overline{\mathscr{R}} \boldsymbol{A}$ defined as $\overline{\mathscr{R} \boldsymbol{A}}$. The correlation operator also relates to the convolution operator by the following formula

$$
\boldsymbol{A} \circledast \boldsymbol{B}=\mathscr{R} \boldsymbol{A} * \boldsymbol{B} .
$$

Now we can properly define a shift-orthogonal matrix. A matrix $\boldsymbol{A}$ is shift-orthogonal if we have the following relation

$$
\boldsymbol{A} \circledast \boldsymbol{A}=\boldsymbol{\Lambda}_{00},
$$

where the matrix $\boldsymbol{\Lambda}_{i j}$ is 1 at location $(i, j)$ and zero elsewhere. It means that all possible shifted copies of $\boldsymbol{A}$ are orthogonal to $\boldsymbol{A}$. Using (16) and expanding the convolution operator with Fourier transforms yields

$$
\begin{aligned}
\mathscr{F} \mathscr{R} \boldsymbol{A} \odot \mathscr{F} \boldsymbol{A} & =\mathscr{F} \boldsymbol{\Lambda}_{00} \\
& =\mathbb{1},
\end{aligned}
$$

where $\mathbb{1}$ is the matrix filled with 1 and $\odot$ is the entry-wise product. Since $\boldsymbol{A}$ is real, we can show that $\mathscr{F} \mathscr{R} \boldsymbol{A}=\overline{\mathscr{F} A}$. So (19) reduces to

$$
\overline{\mathscr{F} \boldsymbol{A}} \odot \mathscr{F} \boldsymbol{A}=\mathbb{1} .
$$

Each entry of $\mathscr{F} \boldsymbol{A}$ is then of modulus 1 . We introduce the so called randomization of the angle that represents the angle of the entries of $\mathscr{F} \boldsymbol{A}$. $\boldsymbol{A}$ can then be written

$$
\boldsymbol{A}=\mathscr{F}^{-1} \boldsymbol{E},
$$

where $\boldsymbol{E}$ is any matrix a complex numbers of modulus 1 that verifies

$$
\mathscr{R} \boldsymbol{E}=\bar{E} .
$$

However, it might seem unnecessarily restrictive to impose all the shifted copies of $\boldsymbol{A}$ to form an orthogonal family especially in our use case where we are only interested in the orthogonality of shifted copies represented by the measurement pattern $E$ (see (8)).

Here is a simple example that illustrates this fact. Suppose that, for some reason, we want our measurement matrix $\boldsymbol{A}$ to sum to 0 . We have

$$
\boldsymbol{A} \circledast \mathbb{1}=0 .
$$

This means that we have a set of $N M+1$ matrices, namely

$$
S_{00}(\boldsymbol{A}), \ldots, S_{N-1, M-1}(\boldsymbol{A}) \text { and } \mathbb{1},
$$

that forms an orthogonal family which is impossible because the matrix $\boldsymbol{A}$ only has $N M$ coefficients. This simple example shows that the classic generation of such matrices is too restrictive. This is especially a concern for our problem that is to find a "random enough" measurement matrix.

In the following, we propose a less restrictive method that forces only the desired shifted copies to form an orthogonal system. We then show that it is possible in this case to apply more constraints.

\section{B. Partial shift-orthogonal matrix}

We are going to relax the shift-orthogonality constraint (17) by the orthogonality of family (8) only. According to (9), it means that for all different $f$ and $g$ in $E$, we want

$$
\operatorname{Tr} S_{f}(\boldsymbol{A})^{T} S_{g}(\boldsymbol{A})=0,
$$

which can be shown to be equivalent to

$$
\operatorname{Tr} S_{f-g}(\boldsymbol{A})^{T} \boldsymbol{A}=0,
$$

which means, according to (9), that

$$
(\boldsymbol{A} \circledast \boldsymbol{A})_{f-g}=0 .
$$

Therefore, we define a new pattern from the measurement pattern $E$, denoted $\widetilde{E}$, defined as follows

$$
\widetilde{E}=\{f-g \mid f, g \in E, f \neq g\} .
$$

The problem (8) is now equivalent to finding a matrix $\boldsymbol{A}$ such that

$$
\boldsymbol{A} \circledast \boldsymbol{A}=\boldsymbol{N},
$$

where $\boldsymbol{N}_{i j}=0$ for all $(i, j) \in \widetilde{E}$. Note that the matrix $\boldsymbol{N}$ has to be $\mathscr{R}$-invariant. If we expand the convolution operator with Fourier transforms, we have

$$
\mathscr{F}^{-1}(\mathscr{F} \mathscr{R} \boldsymbol{A} \odot \mathscr{F} \boldsymbol{A})=\boldsymbol{N} .
$$

The matrix $\boldsymbol{A}$ we want to generate is real. According to (15) and because $\mathscr{F}$ and $\mathscr{R}$ commute, it implies that

$$
\mathscr{F} \mathscr{R} \boldsymbol{A}=\overline{\mathscr{F} \boldsymbol{A}} .
$$

The equality (29) reduces to

$$
|\mathscr{F} \boldsymbol{A}|^{2}=\mathscr{F} \boldsymbol{N} .
$$


We first remark that $\mathscr{F} \boldsymbol{N}$ has to have all its entries nonnegative. Then, it suffices to have

$$
|\mathscr{F} \boldsymbol{A}|=\sqrt{\mathscr{F} \boldsymbol{N}} .
$$

for equality (32) to be verified. There is no constraint on the argument of the coefficients of $\mathscr{F} \boldsymbol{A}$. We just have to make sure that $\mathscr{F} \boldsymbol{A}$ is $\overline{\mathscr{R}}$-invariant. We can then write

$$
\mathscr{F} \boldsymbol{A}=\boldsymbol{E} \odot \sqrt{\mathscr{F} \boldsymbol{N}}
$$

where $\boldsymbol{E}$ is a $\overline{\mathscr{R}}$-invariant matrix of complex numbers of norm 1. Finally, we have

$$
\boldsymbol{A}=\mathscr{F}^{-1}(\boldsymbol{E} \odot \sqrt{\mathscr{F} \boldsymbol{N}}) .
$$

Conversely, all matrices of the form (35) verify the condition (8). This result is summarized in the following theorem.

Theorem 1. The real matrices $\boldsymbol{A}$ such that the family (8) is orthogonal are the matrices that write

$$
\boldsymbol{A}=\mathscr{F}^{-1}(\boldsymbol{E} \odot \sqrt{\mathscr{F} \boldsymbol{N}}),
$$

where $\boldsymbol{E}$ is any $\overline{\mathscr{R}}$-invariant matrix of complex numbers of norm 1 and $N$ any real $\mathscr{R}$-invariant matrix that vanishes at $\widetilde{E}$ and such that all entries of $\mathscr{F} N$ are non-negative.

From equation (30), we can show that

$$
\left(\sum_{i j} \boldsymbol{A}_{i j}\right)^{2}=\sum_{i j} \boldsymbol{N}_{i j} .
$$

For a simple shift-orthogonal measurement matrix we have $\boldsymbol{N}=\boldsymbol{\Lambda}_{00}$ meaning that such a matrix has to sum to \pm 1 . Partial shift-orthogonal matrices do not suffer from such a restriction because the matrix $\boldsymbol{E}$ also has to be specified allowing them to be much more flexible.

\section{Practical example and discussion}

We detail the steps established in the previous section and show the generation of a partial shift-orthogonal matrix. Suppose we want a $5 \times 5$ matrix where the shifted copies represented by the pattern $E$

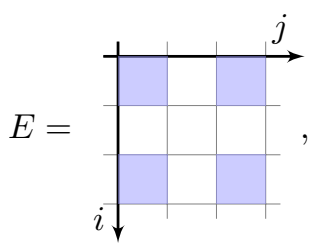

form an orthogonal family. According to theorem 1, we first need to calculate $\widetilde{E}$ according to (28) and its reduced modulo
5, since we are working in $\mathbb{Z}_{5} \times \mathbb{Z}_{5}$,

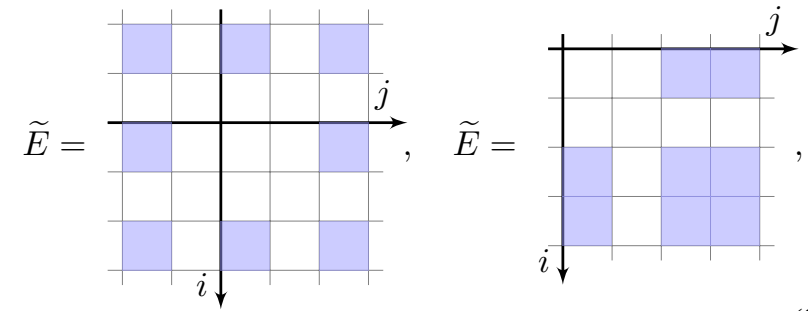

which means that the matrix $N$ looks like this

$$
\boldsymbol{N}=\left(\begin{array}{ccccc}
1 & \star & 0 & 0 & \star \\
\star & \star & \star & \star & \star \\
0 & \star & 0 & 0 & \star \\
0 & \star & 0 & 0 & \star \\
\star & \star & \star & \star & \star
\end{array}\right) .
$$

Then we replace the stars by random inner product values so as to have $\overline{\mathscr{R}} \boldsymbol{N}=\boldsymbol{N}$ and $\mathscr{F} \boldsymbol{N}$ non-negative and apply the formula (36) which yields

$$
\boldsymbol{A}=\left(\begin{array}{ccccc}
-0.15 & -0.02 & -0.16 & 0.14 & 0.14 \\
0.21 & 0.25 & -0.42 & -0.03 & 0.19 \\
0.15 & -0.03 & 0.29 & -0.47 & 0.04 \\
0.11 & -0.08 & -0.07 & -0.04 & -0.08 \\
0.18 & 0.17 & 0.34 & -0.04 & 0.22
\end{array}\right)
$$

We verify that the correlation vanishes at the right locations.

$$
\boldsymbol{A} \circledast \boldsymbol{A}=\left(\begin{array}{ccccc}
1 & -0.09 & 0 & 0 & -0.09 \\
-0.1 & 0.29 & -0.03 & -0.2 & -0.17 \\
0 & 0.03 & 0 & 0 & 0.11 \\
0 & 0.11 & 0 & 0 & 0.03 \\
-0.1 & -0.17 & -0.2 & -0.03 & 0.29
\end{array}\right) .
$$

We are interested in limiting as much as possible the number of elements of $\widetilde{E}$. Indeed, if $\widetilde{E}$ is of maximal size (i.e. $\widetilde{E}=\{(i, j), 0 \leq i, j<n\})$ then the matrix $N$ in equation (40) would be equal to $\boldsymbol{\Lambda}_{00}$ and we would fall back in the classical case seen in section III.

As a consequence, we will restrict ourselves to measurement patterns $E$ that are quasi-rectangular. With this restriction, it is not difficult to see that

$$
|\widetilde{E}| \sim 4 \cdot|E| .
$$

In that case, the pattern $\widetilde{E}$ is basically obtained by replicating $E$ on the three other quadrants, hence the " 4 " in equation (43).

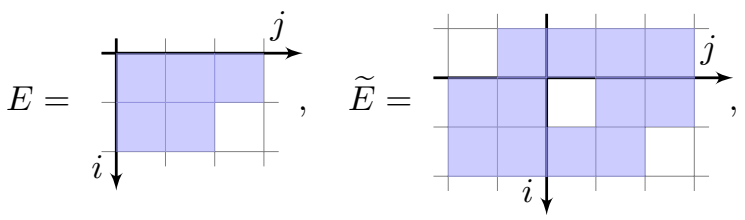

With that equivalence the measurement rate as defined in [20] verifies

$$
\frac{|E|}{N M}<\frac{1}{4},
$$

since we also have $|\widetilde{E}| \leq N M$. As a result, for a partial shift-orthogonal measurement matrix to be more general than 
shift-orthogonal ones we have to take a measurement rate less than $1 / 4$.

\section{NUMERICAL EXPERIMENTS: SIGNAL RECONSTRUCTIONS}

In this section, partial shift-orthogonal matrices are compared with 7 classical measurement matrices in the frame of signal reconstructions [12], [17], [22], [23]:

- Gaussian random matrices (G),

- Toeplitz matrices (T),

- Bernouilli matrices (B),

- circulant matrices whose first row is Gaussian-distributed (CG),

- learning circulant matrices (LC) [17],

- shift-orthogonal matrices (SO),

- partial shift-orthogonal matrices (PSO).

First we propose to reconstruct synthetic signals and then real MRS signals.

\section{A. Reconstruction of synthetic signals}

The first set of experiments is conducted on a random sparse signal of size 512 with 75 spikes. To achieve better precision, for each measurement rate, ranging from 10 to $30 \%$, we solve the minimization problem 100 times. The results (a) $10 \%$

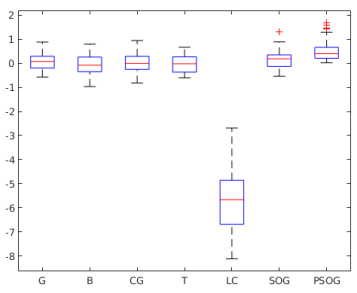

(c) $30 \%$

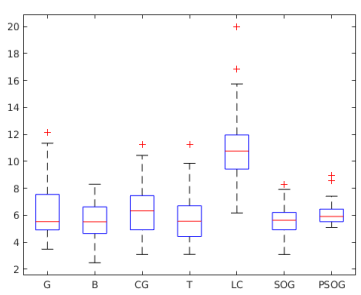

(b) $20 \%$

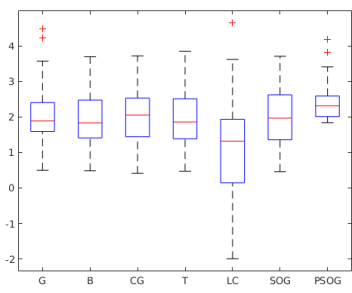

(d) $40 \%$

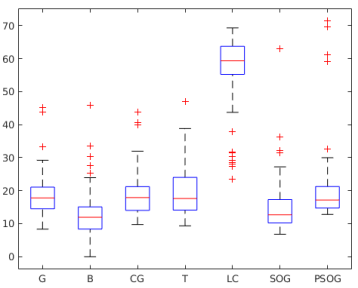

Fig. 2: SNR of reconstruction using 7 different sensing matrices on a sparse signal.

are shown on Fig 2. We obtain better reconstruction results with learning circulant matrices when the measurement rate is high (30 and 40\%). When the rate is low (10 and 20), PSO matrices perform and we obtain better reconstruction median value with low dispersion. The sparsity of signals is finally too high to obtained good reconstruction results with LC matrices. At these rates, we have similar statistical results with Gaussian, Gaussian circulant, Bernouilli and Toeplitz matrices.
The second signal is a sinusoidal signal with a few periods and of size 512. We use a DCT as sparsifying basis. The results are shown in Fig 3. In this framework, the proposed PSO matrices is as efficient as the reference matrices (Gaussian, Bernouilli and circulant Gaussian). We note the PSO matrix

When we decrease the measurement rate, we obtain a result with PSO matrices close to the one with Gaussian matrices (which is our reference). Compared to the Gaussian, Bernouilli and circulant Gaussian matrices, we observe a lower statistical dispersion. (a) $10 \%$

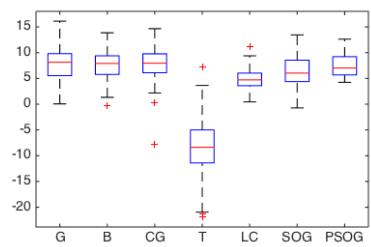

(c) $30 \%$

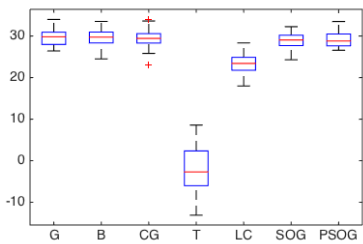

(b) $20 \%$

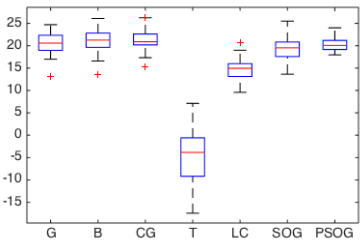

(d) $40 \%$

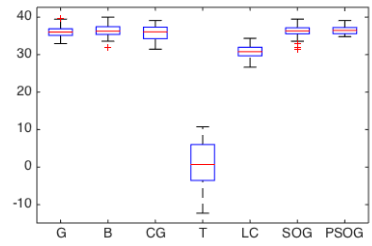

Fig. 3: SNR of sinus reconstructions using 7 different sensing matrices on a sinusoidal signal.

In both experiments, the efficiency of partial shiftorthogonal matrices are between full Gaussian ones and shiftorthogonal ones. Though, the measurement rate should be kept small for the difference to be significant.

\section{B. Reconstruction of real MRS data}

Magnetic Resonance Imaging apply compressive sensing to accelerate the acquisition [24], [25]. We thus proposed to reconstruct in vivo Magnetic Resonance Spectroscopy (MRS) data which were acquired using $3 \mathrm{~T}$ whole-body system (Verio, Siemens, Erlangen, Germany) and were provided by the radiology department of CHU Poitiers (France). We work on 16 signals with a number of samples of signals is equal to 1024.

The range of measurement rate is from 10 to $40 \%$ and we solve the minimization problem 100 times on the signal. On Fig. 4 PSO matrices provide best reconstructions with a rate equal to $20 \%$ and $30 \%$. When the measurement rate is low, Toeplitz matrices have a better median value (3.02 at $10 \%)$. Reconstructions with PSO matrices provide the second best result (2.60) with less statistical dispersion. When the measurement rate is high (superior to 40\%) the learning circulant matrices obtained best results with a high median value and with less dispersion. Note that Bernouilli matrix is not adapted to this signal reconstruction. 
(a) $10 \%$

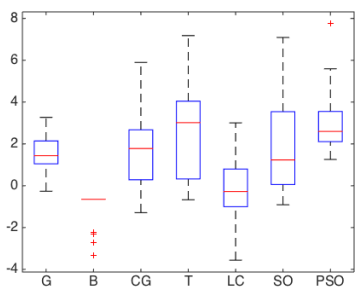

(c) $30 \%$

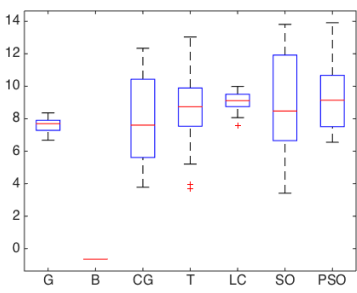

(b) $20 \%$

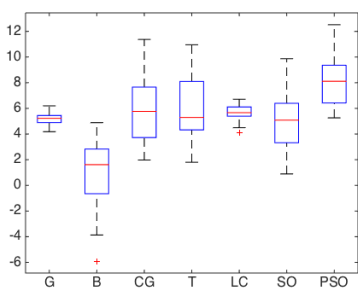

(d) $40 \%$

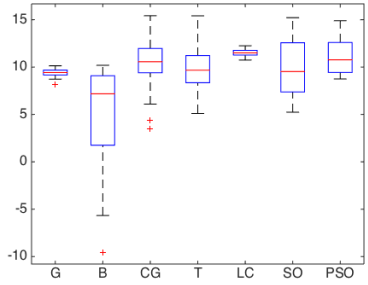

(a) MRS signal

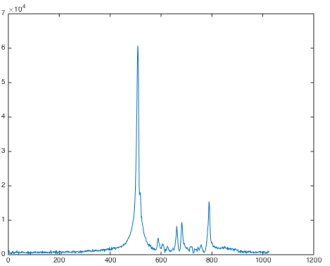

(c) $\mathrm{CG}$

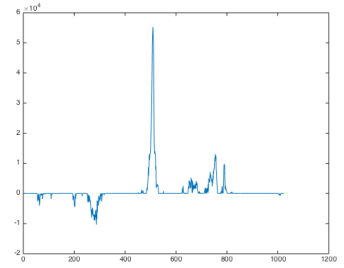

(e) LC

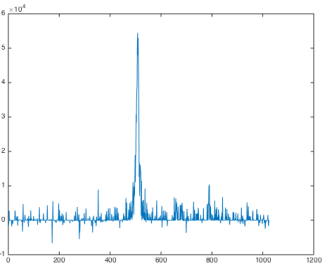

(b) $\mathrm{G}$

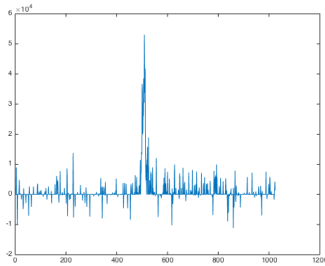

(d) $\mathrm{T}$

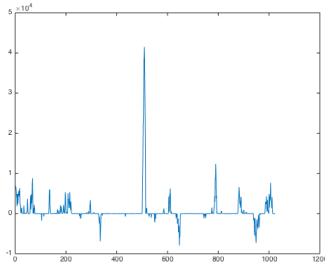

(f) $\mathrm{SO}$

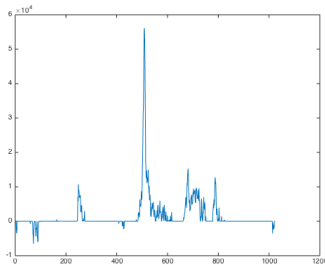

(g) PSO

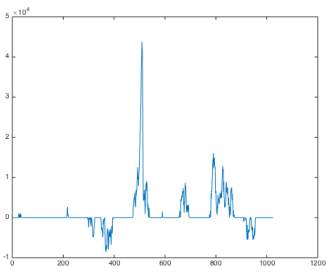

Fig. 5: Signal reconstruction using different sensing matrices on MRS data with a measurement rate equal to $20 \%$.

We will discuss the pattern detection results with different measurement matrices, measurement rates and color patterns.

\section{B. Compressive pattern matching}

1) Pattern detections with different sensing matrices: We test our algorithm on a publicly available color image of Giza, Egypt. We extract a $128 \times 128$ image shown in Fig. $6 \mathrm{~b}$ and we want to detect the locations where there is sand surrounded by vegetation. We test the detection of patterns which are displayed in Fig. 6 a.

Fig. 7 illustrates the detections of pattern 2. We obtained these results by using thresholding; the threshold is empirically set at 80 percent from the maximum value. The results are confirming our previous observations. Even if there is a small difference between shift-orthogonal matrices and fully

\section{interested in form an orthogonal family.}

We describe the algorithm of pattern detection on an image after a compressive sensing in Algorithm 1.

In this applicative framework, we propose to generate a pattern dependent circulant matrix whose only rows we are 


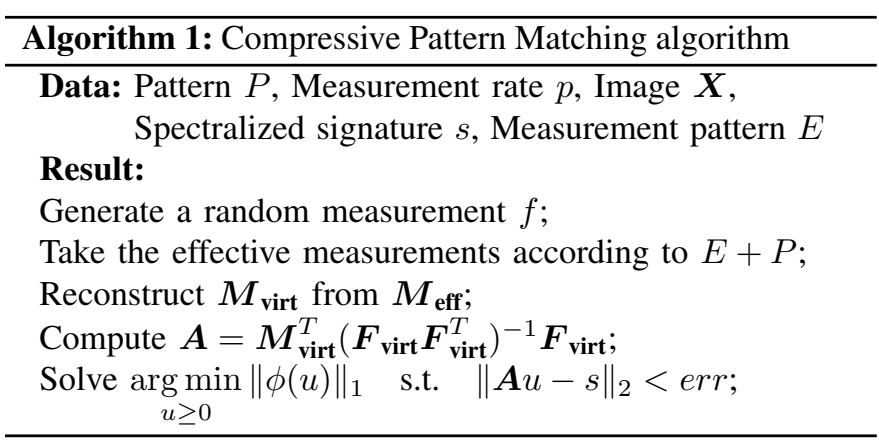

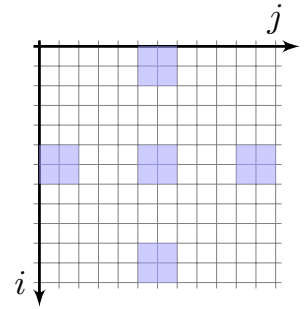

(a)

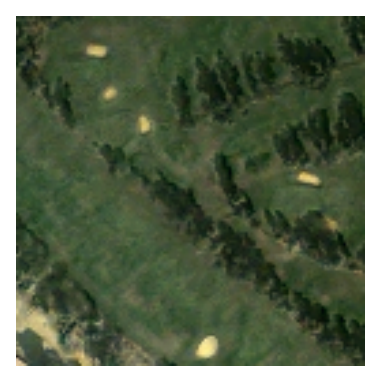

(b)
Fig. 6: (a) Proposed pattern; (b) Giza, Egypt image.

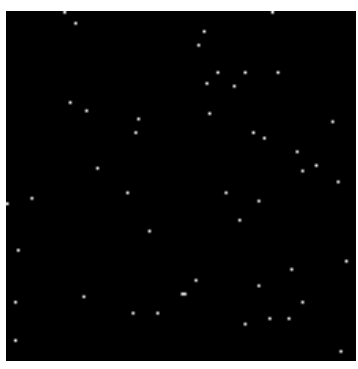

(a) Gaussian

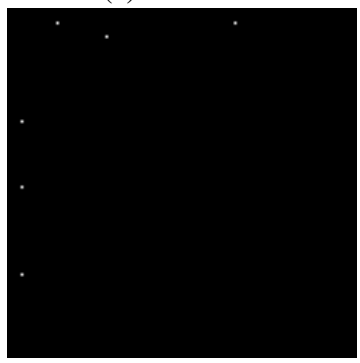

(c) Toeplitz

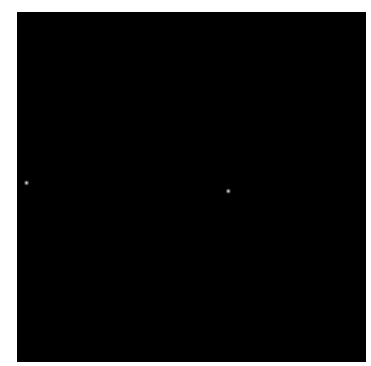

(b) Bernouilli

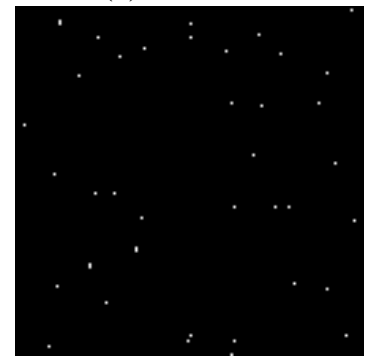

(d) shift-orthogonal

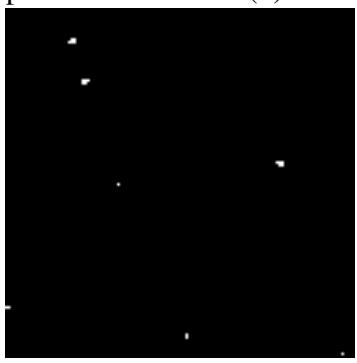

(e) partial shift-orthogonal

Fig. 7: Detection of patterns with the 6 different sensing matrices.
Gaussian ones, we can still improve the detection by choosing partial shift-orthogonal sensing matrices. Results with the Toeplitz matrices, circulant Gaussian matrices and are too bad and the patterns are not detected (see the result with a Toeplitz matrix on Fig. 7c).

We solve the minimization problem 100 times for each sensing matrix. We set the measurement rate to $20 \%$. In Tab. I, we observe a better the partial shift-orthogonal matrices obtain better results than the full Gaussian and shift-orthogonal matrices. We detect in average 2.5 false patterns.

\begin{tabular}{c|c|c} 
Full Gaussian & SOG & PSOG \\
\hline $75 \%$ & $74 \%$ & $80 \%$
\end{tabular}

TABLE I: Average number of detection errors for three different sensing matrices with a measurement rate set to $20 \%$.

We test the compressive pattern detection using Gaussian, shift-orthogonal and partial shift-orthogonal matrices. We obtain a relatively low computational time equal to 670 seconds with a partial shift-orthogonal matrix (we use Matlab on Linux with Quad-core Intel Core i7-6820HQ). The computational time with a Gaussian matrix is 7850 seconds or more than 10 times slower. The computer is however not enough efficient to test the pattern detections with learning circulant matrices.

2) Pattern detections with different measurement rates: We test our algorithm on the color image named girl. We extract a $128 \times 128$ image shown in Fig. 10b and we want to detect the locations of the blue pearl. The aim is to validate that a color can be detected from a compressive sensing of a color image.

As shown on Fig. $8 b$ we extract the blue value on the original image and construct a square. We test the detection of this pattern (see on Fig. 8a).

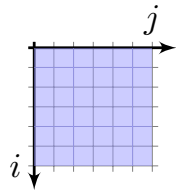

(a)

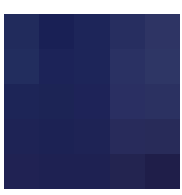

(b)

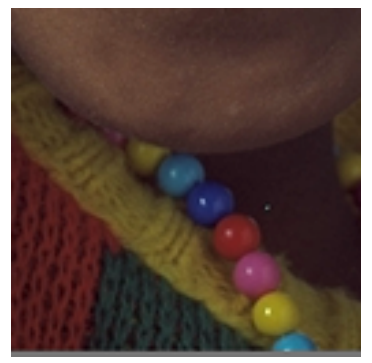

(c)
Fig. 8: (a) Proposed pattern; (b) a part of the blue pearl; (b) a part of image Girl.

Fig. 9 shows that we detect correctly the blue pearl with the three different measurement rates but we add false detection when we decrease the rate.

3) Color edge detection: On image 10b we want to detect the transition between the yellow and green and we set the measurement rate to $15 \%$. The aim is to validate that color edges can be detected from a compressive sensing of a color image.

We use the pattern which are displayed on Fig. 10a and the partial shift-orthogonal sensing matrix. 


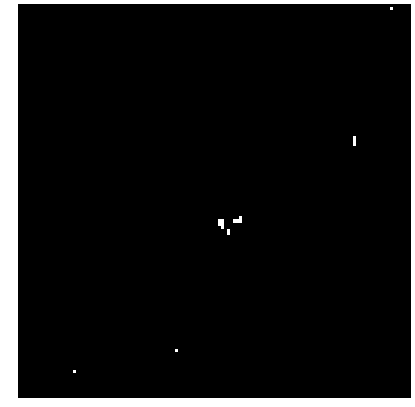

(a) $15 \%$

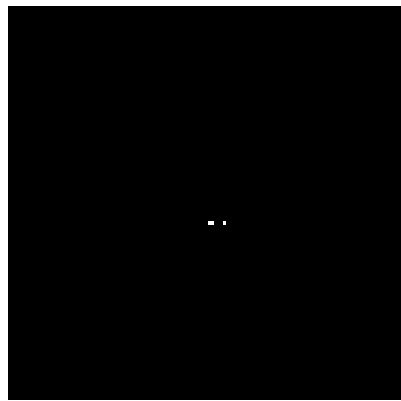

(b) $20 \%$

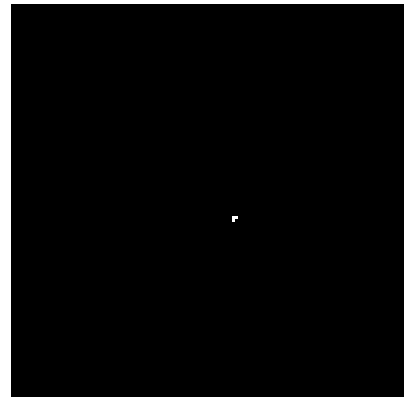

(c) $25 \%$

Fig. 9: Pattern detection with partial shift-orthogonal measurement matrices and 3 different measurement rates.

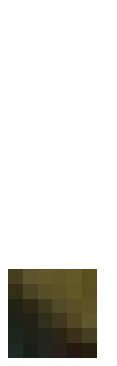

(a)

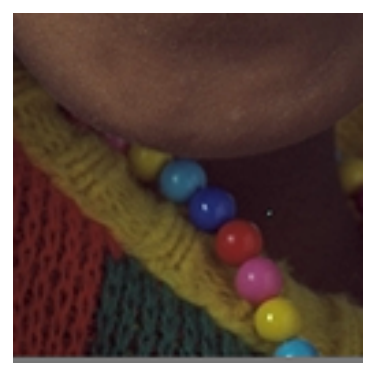

(b)

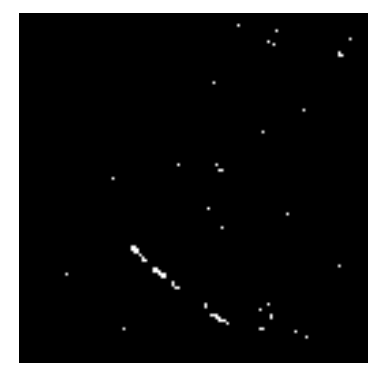

(c)
Fig. 10: Color edge detections with $15 \%$ of measurements: (a) the pattern; (c) detection result.

Fig. 10c shows that we detect correctly the transition between the green texture and the yellow one with a low measurement rate. The result is correct and we observe that the transition is not detected when the orientation is not the same as the pattern.

\section{Conclusion And PeRspectives}

This paper introduces an improved way to generate circulant orthogonal or shift-orthogonal sensing matrices that more precisely fit the minimization problem's need. Our hope was that a more relaxed construction of such matrices would yield better sensing matrices. Partial shift-orthogonal matrices are tested in different scenarii: the first two on one-dimensional signals, a reconstruction of a MRS signal and two pattern detections on color images with minimization problem developed in a previous paper. We have compared the proposed sensing matrix with different sensing matrices in a framework of compressive reconstruction and we have illustrated the obtained quality of reconstructions. We have shown that the partial shift-orthogonal matrix allows to detect a pattern on multispectral images with few bands as a color images. We have also shown that we can detect a color patch and the edges between two colors from a compressive sensing of a color image.

Even if the difference between full Gaussian sensing matrices and shift-orthogonal ones is small, the efficiency of partial shift-orthogonal measurement matrices lies between the two. The new generation process only requires one more Fourier transform as opposed to the shift-orthogonal case.

A further development would be to look if we can losen the constraints even more and drop the partial orthogonality constraint. Indeed, we are able to specify the value of the inner product between the measurement matrix and a given shifted copy of it. We have set this value to zero to have an orthogonality property but nothing prevents us from choosing a non-zero value. The open question is how to choose those values so as to have a measurement matrix that would still enjoy a nice structure allowing us to quickly compute the product with its transpose.

\section{APPENDIX A}

\section{MATRIX DEFINITION FOR RECONSTRUCTION USING SHIFTED MEASUREMENTS [20]}

Let $\boldsymbol{B}$ be the measurement matrix that is the 2-dimensional version of the measurement vector $f$. We then have

$$
\operatorname{vec}(\boldsymbol{B})=f .
$$

If the measurement matrix $\boldsymbol{B}$ is shifted by a vector $e$, it becomes $S_{e}(\boldsymbol{B})$. The shifted measurement vector $f_{e}$ is then

$$
f_{e}=Q_{e} f
$$

Suppose all our virtual measurements are a shifted version of one measurement $f$, the measurements writes

$$
m_{e}=f^{T} \boldsymbol{Q}_{-e} \operatorname{spec}_{P}(\boldsymbol{X})
$$

The shifting information is modelled as a subset $E=$ $\left\{e_{1}, \ldots, e_{|E|}\right\}$ of $\mathbb{Z}^{2}$ and the virtual measurement matrix is defined by

$$
\boldsymbol{M}_{\text {virt }}=\boldsymbol{F}_{\text {virt }} \operatorname{spec}_{P}(\boldsymbol{X})
$$

with

$$
\boldsymbol{M}_{\mathbf{v i r t}}=\left(\begin{array}{c}
m_{e_{1}} \\
\vdots \\
m_{e_{|E|}}
\end{array}\right) \quad \text { and } \quad \boldsymbol{F}_{\text {virt }}=\left(\begin{array}{c}
f^{T} \\
f^{T} \boldsymbol{Q}_{-e_{2}} \\
\vdots \\
f^{T} \boldsymbol{Q}_{-e_{|E|}}
\end{array}\right)
$$

To reconstruct $M_{\text {virt }}$ that gathers the results of measurements on the virtual image $\operatorname{spec}_{P}(\boldsymbol{X})$, we need to take the shifted measurements represented by $E^{\prime}=E+P$ where $E+P$ denotes the set

$$
\left\{e_{i}+p_{j}\left|e_{i} \in E, 1 \leq i \leq\right| E\left|, p_{j} \in P, 1 \leq j \leq\right| P \mid\right\} .
$$


Therefore, we choose

$$
\boldsymbol{F}_{\text {eff }}=\left(\begin{array}{c}
f^{T} \\
f^{T} \boldsymbol{Q}_{-e_{2}^{\prime}} \\
\vdots \\
f^{T} \boldsymbol{Q}_{-e_{\left|E^{\prime}\right|}^{\prime}}
\end{array}\right),
$$

and the effective sensing matrix is obtained by

$$
M_{\text {eff }}=F_{\text {eff }} X
$$

\section{REFERENCES}

[1] M. F. Duarte, M. A. Davenport, D. Takhar, J. N. Laska, T. Sun, K. F. Kelly, and R. G. Baraniuk, "Single-pixel imaging via compressive sampling," IEEE signal processing magazine, vol. 25, no. 2, pp. 83-91, 2008.

[2] D. Takhar, J. N. Laska, M. B. Wakin, M. F. Duarte, D. Baron, S. Sarvotham, K. F. Kelly, and R. G. Baraniuk, "A new compressive imaging camera architecture using optical-domain compression," in Electronic Imaging 2006. International Society for Optics and Photonics, 2006, pp. 606 509-606509.

[3] M. B. Wakin, J. N. Laska, M. F. Duarte, D. Baron, S. Sarvotham, D. Takhar, K. F. Kelly, and R. G. Baraniuk, "An architecture for compressive imaging," in Image Processing, 2006 IEEE International Conference on. IEEE, 2006, pp. 1273-1276.

[4] M. Wakin, J. N. Laska, M. F. Duarte, D. Baron, S. Sarvotham, D. Takhar, K. F. Kelly, and R. G. Baraniuk, "Compressive imaging for video representation and coding," in Picture Coding Symposium, vol. 1, 2006, p. 13.

[5] J. Romberg, "Imaging via compressive sampling [introduction to compressive sampling and recovery via convex programming]," IEEE Signal Processing Magazine, vol. 25, no. 2, pp. 14-20, 2008.

[6] E. Candès, J. Romberg, and T. Tao, "Stable signal recovery from incomplete and inaccurate measurements," Communications on pure and applied mathematics, vol. 59, no. 8, pp. 1207-1223, 2006.

[7] D. Donoho, "Compressed sensing," Information Theory, IEEE Transactions on, vol. 52, no. 4, pp. 1289-1306, 2006.

[8] E. Candes and J. Romberg, "Sparsity and incoherence in compressive sampling," Inverse problems, vol. 23, no. 3, p. 969, 2007.

[9] J. Romberg, "Compressive sensing by random convolution," SIAM Journal on Imaging Sciences, vol. 2, no. 4, pp. 1098-1128, 2009.

[10] W. U. Bajwa, J. D. Haupt, G. M. Raz, S. J. Wright, and R. D. Nowak, "Toeplitz-structured compressed sensing matrices," in Statistical Signal Processing, 2007. SSP'07. IEEE/SP 14th Workshop on. IEEE, 2007, pp. 294-298.

[11] H. Rauhut, J. Romberg, and J. A. Tropp, "Restricted isometries for partial random circulant matrices," Applied and Computational Harmonic Analysis, vol. 32, no. 2, pp. 242-254, 2012.

[12] H. Rauhut, "Circulant and toeplitz matrices in compressed sensing," arXiv preprint arXiv:0902.4394, 2009.

[13] W. Yin, S. Morgan, J. Yang, and Y. Zhang, "Practical compressive sensing with toeplitz and circulant matrices," in Visual Communications and Image Processing 2010. International Society for Optics and Photonics, 2010, pp. $77440 \mathrm{~K}-77440 \mathrm{~K}$.

[14] E. J. Candes and T. Tao, "Decoding by linear programming," IEEE transactions on information theory, vol. 51, no. 12, pp. 4203-4215, 2005.

[15] M. Elad, "Optimized projections for compressed sensing," Signal Processing, IEEE Transactions on, vol. 55, no. 12, pp. 5695-5702, 2007.

[16] J. M. Duarte-Carvajalino and G. Sapiro, "Learning to sense sparse signals: Simultaneous sensing matrix and sparsifying dictionary optimization," Image Processing, IEEE Transactions on, vol. 18, no. 7, pp. 1395-1408, 2009.

[17] Y. Xu, W. Yin, and S. Osher, "Learning circulant sensing kernels," DTIC Document, Tech. Rep., 2014.

[18] M. Pereira, L. Lovisolo, E. da Silva, and M. de Campos, "On the design of maximally incoherent sensing matrices for compressed sensing using orthogonal bases and its extension for biorthogonal bases case," Digital Signal Processing, vol. 27, pp. 12-22, 2014.

[19] Z. Guo and S. Osher, "Template Matching via L1 Minimization and Its Application to Hyperspectral Data," Inverse Problems and Imaging, vol. 5, no. 1, pp. 19-35, 2011.
[20] S. Rousseau, D. Helbert, P. Carré, and J. Blanc-Talon, "Compressive pattern matching on multispectral data," Geoscience and Remote Sensing, IEEE Transactions on, vol. 52, no. 12, 2014. [Online]. Available: arXiv preprint arXiv:1403.6958

[21] S. Rousseau, D. Helbert, P. Carré, and J. Blanc-Talon, "Compressive template matching on multispectral data," in International Conference on Acoustics, Speech, and Signal Processing (ICASSP). Vancouver, Canada: IEEE, 2013.

[22] A. Moshtaghpour, L. Jacques, V. Cambareri, K. Degraux, and C. De Vleeschouwer, "Consistent basis pursuit for signal and matrix estimates in quantized compressed sensing," IEEE signal processing letters, vol. 23, no. 1, pp. 25-29, 2016.

[23] S. Dirksen, H. C. Jung, and H. Rauhut, "One-bit compressed sensing with partial gaussian circulant matrices," arXiv preprint arXiv:1710.03287, 2017.

[24] Y. Yu, J. Jin, F. Liu, and S. Crozier, "Multidimensional compressed sensing mri using tensor decomposition-based sparsifying transform," PloS one, vol. 9, no. 6, p. e98441, 2014.

[25] K. Arai, C. Belthangady, H. Zhang, N. Bar-Gill, S. DeVience, P. Cappellaro, A. Yacoby, and R. L. Walsworth, "Fourier magnetic imaging with nanoscale resolution and compressed sensing speed-up using electronic spins in diamond," Nature nanotechnology, vol. 10, no. 10, p. 859, 2015. 